\title{
Modelling the Double Peak Phenomenon
}

\author{
Godfrey KR*, Arundel PA, Zhu W, Dong Z and Bryant R
}

School of Engineering, University of Warwick, Coventry CV4 7AL, UK

\begin{abstract}
Multiple peaking in concentration-time profiles following the administration of a single dose of a drug has been the subject of considerable interest recently. Two methods of modelling the phenomenon of Double Peaks in pharmacokinetics, both based on compartmental models were described in a recent paper. The first method, the Variability of Absorption approach, assumes that the absorption of the drug from the gut to the systemic plasma varies with the location of the drug in the gut, with negligible absorption through the jejunum and constant (but not necessarily the same) absorption rates in the duodenum and the ileum. The second method, the Parallel Inputs approach, assumes simultaneous input via two parallel pathways. In the present paper the emphasis is on improved fitting of the tail-end of the profiles for both approaches, especially at low plasma concentrations, by use of different forms of data weighting, or alternatively by introducing more complexity in their distribution models. The two modelling approaches are used to model four data sets from the literature with the measurement in each case being concentration of drug in the systemic plasma following a single oral dose of the drug, either in solution form or as an emulsion.
\end{abstract}

Keywords: Double peak; Oral administration; Pharmacokinetics; Single dose

\section{Introduction}

Multiple peaking in concentration-time profiles following the administration of a single dose of a drug has been the subject of considerable interest recently, and a comprehensive review of the possible causes and the associated literature has been published recently [3]. For most drugs, the plasma concentration-time profile following extravascular administration of a single dose rises to a peak and then decays away again, but for some drugs, the profile rises to a peak, starts to decay, then rises again to a second peak, often higher than the first. This is known as the Double Peak Phenomenon.

The present paper is concerned with modelling this phenomenon in profiles following oral doses of drug. The absorption of drug into the blood stream is a very complex process which is affected by many factors $[3,4]$. Three main explanations have been advanced in the literature for this Double Peak Phenomenon, namely enterohepatic recirculation [12], delayed gastric emptying $[10,7,11,8]$, and variability of absorption $[14,6,16,13,15]$.

For the veralipride responses examined in Godfrey et al. [4], the paper from which the data sets were drawn [14] pointed to variability of absorption as being by far the most probable physiological explanation for the Double Peak Phenomenon. This was also the case for the two additional sets of data drawn from the literature considered in the present paper - danazol responses [2] and ranitidine responses [16] and it was therefore used as the basis for the first modelling approach developed therein, referred to as the Variability of Absorption approach.

The problem with this technique is that it needs several parameters to describe the input function, so that the total number of parameters to be estimated is comparatively large. This means that numerous starting values (initial guesses) need to be used for the estimation, to be reasonably certain that the global minimum has been reached, rather than a local minimum. To overcome this, a second approach was developed, based on a suggestion of Jacquez [5], who noted that a single linear chain of compartments had been used to model system responses in several different application areas and could be used in pharmacokinetics as a method of modelling single peak responses.
For the Double Peak Phenomenon, this was extended to model the absorption phase as two parallel linear chains of compartments connected together in series, with the outputs of each chain added together to form the input to a compartmental model describing the distribution and elimination of the drug [4]. This is referred to as the Parallel Inputs approach.

The two approaches were used in Godfrey et al. [4] to model three sets of data, two provided by AstraZeneca and the third, describing the pharmacokinetics of veralipride, drawn from the literature [14]. The curve fitting was done in Berkeley Madonna [1] with the data unweighted. Rosenbrock's Stiff Algorithm was used because of the wide spread of dynamics. For two of the veralipride responses, the tail end of the data was not fitted very well. Accuracy of the pharmacokinetics at this point in the profile is important because, firstly the terminal slope can indicate whether a drug would accumulate on multiple dosing and secondly because it can control the quality of predicting the efficacy of a drug in a PK/PD model. Two methods of improving the tail end fit for these responses are examined in the present paper. The first method involves extending the Parallel Inputs model by adding a further compartment in the part of the model describing distribution and elimination, while in the second method, the tail end data points are weighted before curve fitting. Two further data sets from the literature, describing the pharmacokinetics of danazol [2] and of ranitidine [16] are also examined in the present paper.

It is found that for most of the responses, it is possible to reduce the Sum of Squared Error considerably by using either or both of

${ }^{*}$ Corresponding author: Keith R. Godfrey, School of Engineering, University of Warwick, Coventry CV4 7AL, U.K. Tel: +44(0)2476 523144; Fax: +44(0)2476 418922; E-mail: K.R.Godfrey@warwick.ac.uk

Received February 01, 2011; Accepted July 25, 2011; Published July 27, 2011

Citation: Godfrey KR, Arundel PA, Zhu W, Dong Z, Bryant R (2011) Modelling the Double Peak Phenomenon. J Bioequiv Availab 3: 101-107. doi:10.4172/ jbb.1000068

Copyright: @ 2011 Godfrey KR, et al. This is an open-access article distributed under the terms of the Creative Commons Attribution License, which permits unrestricted use, distribution, and reproduction in any medium, provided the original author and source are credited. 
Citation: Godfrey KR, Arundel PA, Zhu W, Dong Z, Bryant R (2011) Modelling the Double Peak Phenomenon. J Bioequiv Availab 3: 101-107. doi:10.4172/jbb.1000068

these methods. However, it does not seem possible to predict from examination of the data which method will yield the lowest error in any given case.

Specialist software has been developed to model the Double Peak Phenomenon [9] using a method that effectively models the two peaks separately. It has several differences from the work described in the present paper. Firstly, each part of the profile is divided into a region of absorption only before the peak and elimination only after the peak, which does not seem to relate to the real situation where elimination starts as soon as the drug reaches the liver or the kidneys, and not after delays of several hours. Secondly, it appears from examination of their Equation (1) that the initial concentration in the blood stream is nonzero. Thirdly, the curve stripping procedure applied to the first peak is often done on the basis of a small number of data points. Finally, the two phases of the model have different elimination rate constants; this implies two distinct enzyme systems of relevance in the liver, a most unusual preliminary assumption.

\section{Materials and Methods}

\section{Variability of absorption models}

In this approach, the pharmacokinetics was modelled using either of the compartmental models shown in Figure 1. For both models, Compartment $\mathrm{A}$ is the absorption compartment while Compartment 1 is assumed to contain the systemic plasma in which the concentrationtime profile is measured. In both models, the absorption process and the elimination process are assumed to be first-order.

For Model VA(1), the differential equations for the quantities $x_{\mathrm{A}}$ in Compartment $\mathrm{A}$ and $x_{1}$ in Compartment 1 (assumed to contain the plasma) are given by:

$$
\begin{aligned}
\dot{x}_{\mathrm{A}} & =-k_{\mathrm{a}} x_{\mathrm{A}}+u(t) ; & x_{\mathrm{A}}(0) & =0 \\
\dot{x}_{1} & =k_{\mathrm{a}} x_{\mathrm{A}}-k_{1 \mathrm{e}} x_{1} ; & x_{1}(0) & =0
\end{aligned}
$$

While the corresponding equations for Model VA(2) are given by:

$$
\begin{array}{rlrl}
\dot{x}_{\mathrm{A}}=-k_{\mathrm{a}} x_{\mathrm{A}}+u(t) ; & x_{\mathrm{A}}(0)=0 \\
\dot{x}_{1}=k_{\mathrm{a}} x_{\mathrm{A}}-\left(k_{1 \mathrm{e}}+k_{12}\right) x_{1}+k_{21} x_{2} ; & & x_{1}(0)=0 \\
\dot{x}_{2}=k_{12} x_{1}-k_{21} x_{2} ; & & x_{2}(0)=0
\end{array}
$$

Measurement is of the concentration $x_{1} / V_{1}$, with the same volume of distribution $V_{1}$ being used in both models.

The variability of absorption is modelled by using an input rate $u(t)$ with the shape shown in shown in Figure 2.

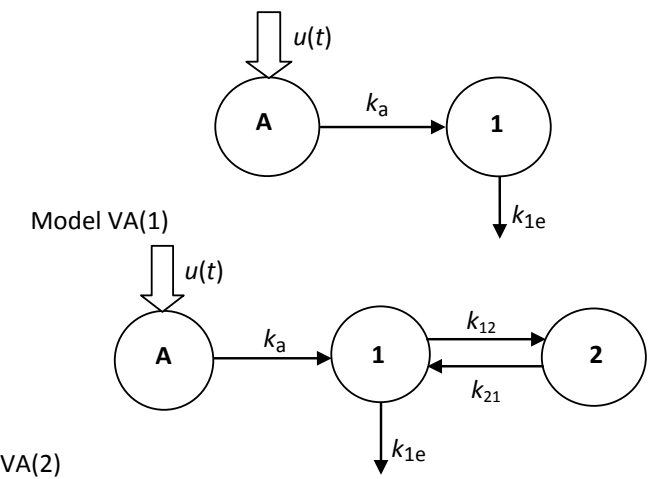

Figure 1: Compartmental models with first-order absorption.

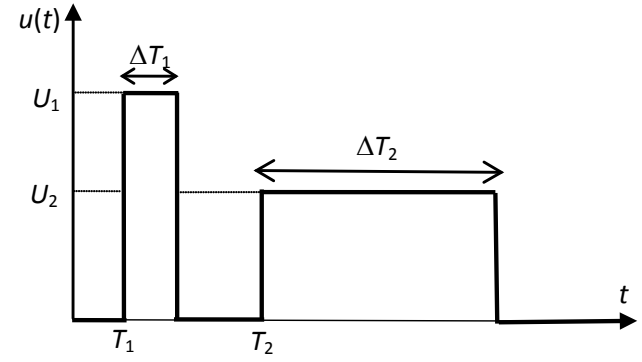

Figure 2: Input rate for two zones of absorption.

There are eight parameters to be estimated for Model VA(1), namely $k_{\mathrm{a}}$ and $k_{1 \mathrm{e}}$ (Equation (1)) and $T_{1}, \Delta T_{1}, U_{1}, T_{2}, \Delta T_{2}$ and $U_{2}$ (Figure 2) while for Model VA(2), there are ten, namely $k_{\mathrm{a}}, k_{1 \mathrm{e}}, k_{12}$ and $k_{21}$ (Equation (2)) and $T_{1}, \Delta T_{1}, U_{1}, T_{2}, \Delta T_{2}$ and $U_{2}$ (Figure 2). The large number of parameters required provided the motivation for the development of a second approach which reduced the number of parameters to be estimated by a third.

\section{Parallel inputs model}

As noted in the Introduction, the second approach developed is based on modelling the absorption phase as two parallel linear chains [4], as shown in Figure 3.

The inputs to both chains are realistically assumed to be impulsive, $D_{1} \delta(t)$ to the first chain and $D_{2} \delta(t)$ to the second. The first chain is designed to model the first peak, and contains a number $N_{1}$ of identical compartments, each with absorption rate constant $k_{\mathrm{al}}$. The second pathway, designed to model the second peak, contains a larger number $\mathrm{N}_{2}$ of identical compartments, each with absorption rate constant $k_{\mathrm{a} 2}$. For Model PI(1), which has a single compartment to describe the distribution and elimination of the drug, the differential equations for the quantities in each compartment are given by

$$
\begin{aligned}
& \dot{x}_{\mathrm{a} 1}=-k_{\mathrm{a} 1} x_{\mathrm{a} 1} ; \\
& \dot{x}_{\mathrm{a} 2}=k_{\mathrm{a} 1} x_{\mathrm{a} 1}-k_{\mathrm{a} 1} x_{\mathrm{a} 2} ; \\
& \vdots \quad \vdots \quad \vdots \\
& \dot{x}_{\mathrm{aN}_{1}}=k_{\mathrm{a} 1} x_{\mathrm{a}\left(\mathrm{N}_{1}-1\right)}-k_{\mathrm{a} 1} x_{\mathrm{aN}_{1}} ; \\
& x_{\mathrm{a} 1}(0)=D_{1} \\
& x_{\mathrm{a} 2}(0)=0 \\
& \dot{x}_{\mathrm{A} 1}=-k_{\mathrm{a} 2} x_{\mathrm{A} 1} \text {; } \\
& \dot{x}_{\mathrm{A} 2}=k_{\mathrm{a} 2} x_{\mathrm{A} 1}-k_{\mathrm{a} 2} x_{\mathrm{A} 2} \text {; } \\
& \vdots \quad \vdots \quad \vdots \\
& \dot{x}_{\mathrm{AN}_{2}}=k_{\mathrm{a} 2} x_{\mathrm{A}\left(\mathrm{N}_{2}-1\right)}-k_{\mathrm{a} 2} x_{\mathrm{AN}_{2}} ; \\
& x_{\mathrm{aN}_{1}}(0)=0 \\
& x_{\mathrm{A} 1}(0)=D_{2} \\
& x_{\mathrm{A} 2}(0)=0 \\
& \dot{x}_{1}=k_{\mathrm{a} 1} x_{\mathrm{aN}_{1}}+k_{\mathrm{a} 2} x_{\mathrm{AN}_{2}}-k_{1 \mathrm{e}} x_{1} \text {; } \\
& x_{\mathrm{AN}_{2}}(0)=0 \\
& x_{1}(0)=0
\end{aligned}
$$

The procedure adopted was to perform the parameter estimation for a large number of combinations of $N_{1}$ and $N_{2}$, leaving only five parameters to be estimated, namely $D_{1}, D_{2}, k_{\mathrm{a} 1}, k_{\mathrm{a} 2}$, and $k_{1 \mathrm{e}}$

For Model PI(2), which has two compartments to describe distribution and elimination, the last of the equations in Equation Set (3) is now replaced with

$$
\begin{array}{lr}
\dot{x}_{1}=k_{\mathrm{a} 1} x_{\mathrm{aN}}+k_{\mathrm{a} 2} x_{\mathrm{AN}_{2}}-\left(k_{12}+k_{1 \mathrm{e}}\right) x_{1}+k_{21} x_{2} ; x_{1}(0)=0 \\
\dot{x}_{2}=k_{12} x_{1}-k_{21} x_{2} ; & x_{2}(0)=0
\end{array}
$$




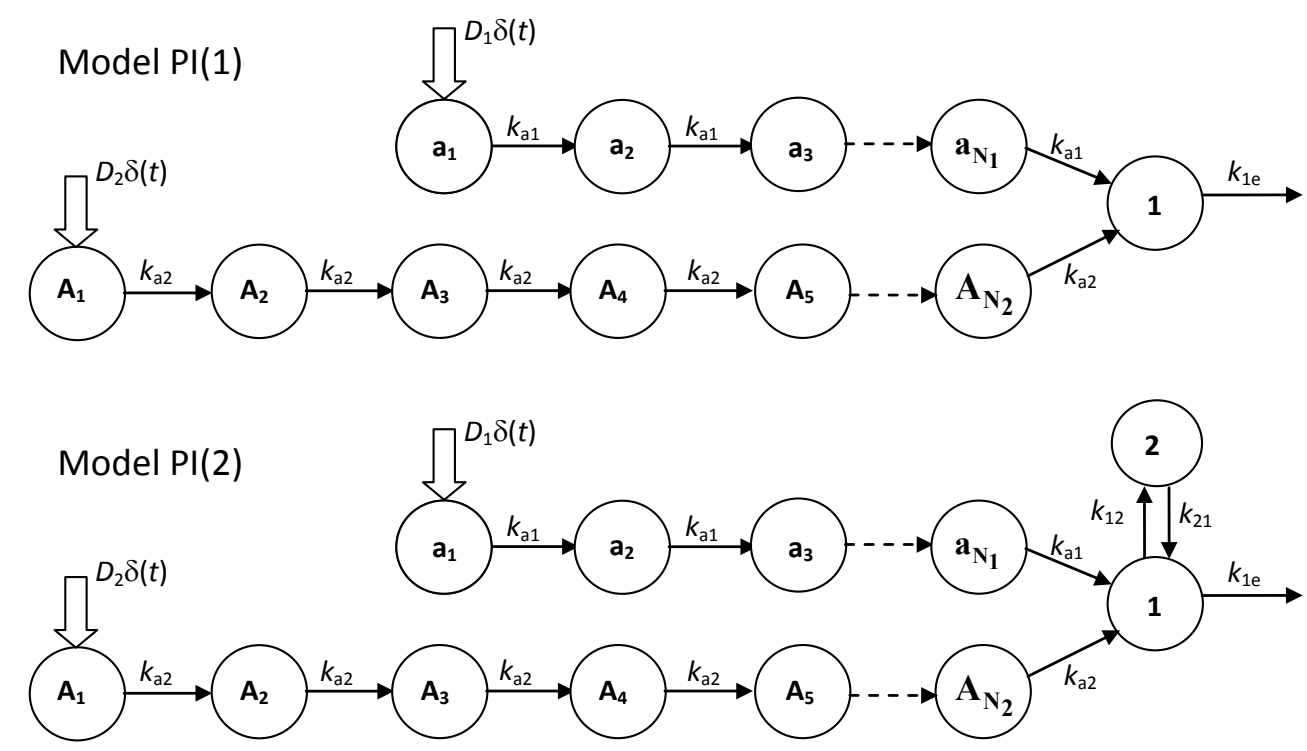

Figure 3: The Parallel Inputs Models $\mathrm{PI}(1)$ and $\mathrm{PI}(2)$.

There are now seven parameters to be estimated - the five above, plus $k_{12}$ and $k_{21}$.

Although the overall parameter estimation procedure can be lengthy with this approach there is compensation in that the initial values chosen for the parameters are considerably less critical to the estimation than for the Variability of Absorption Approach, due to the much smaller number of parameters to be estimated [4].

In both approaches, if suitable plasma concentration data are available following intravenous (IV) dosing of compound then the size of the appropriate distribution models will be known $(1,2$ or even 3 -compartment). Simultaneous fitting of IV and oral data may then be possible which will increase the robustness of the parameters leading to better estimates of bioavailability for profiles exhibiting a double peak.

\section{Data sets}

Four sets of data are examined in this paper; all are concentrationtime profiles following a single orally-administered dose at time $t=$ 0 . Data Set 1 (Table 1) is a subset of the responses for veralipride [14] which were examined earlier [4]. For the first two responses (Subjects $\mathrm{F}$ and $\mathrm{G}$ ), the tail end is not fitted well. It is fitted well for the third response (Subject C), which is included in the present study to see whether there is any improvement in fitting data where the fit to the tail end is already good. The second and third data sets (Tables 2 and 3) consist of three responses for danazol [2] and two responses for ranitidine [16], respectively. These were not examined previously and are included here as further examples of fitting the Double Peak Phenomenon. The fourth set of data (Table 4) is again a subset of the responses examined in Godfrey et al. [4], and consists of a response from a set of data provided by AstraZeneca.

\section{Results}

For all of the model fits, parameters were estimated using Rosenbrock's Stiff Algorithm in the curve fitting procedure in the software package Berkeley Madonna, Version 8.0.1 [1]. The results presented are of normalised RMS Errors, obtained by dividing the
RMS Error by the mean of the concentration values; this gives a better comparison between subjects.

For the Variability of Absorption modelling, because of the many parameters to be estimated, it was necessary to make a very large number of fits with different initial guesses for the parameter values in order to be reasonably certain that the global minimum in RMS Error was reached. The number of fits used throughout this work was set to 500 .

As stated earlier, for the Parallel Inputs modelling, the number of compartments $N_{1}$ and $N_{2}$ in each chain were not among the parameters to be estimated using Berkeley Madonna and instead, the fitting was repeated for a large number of these integer values.

The Normalised RMS Errors obtained with no weighting of the data are given in Table 5 and the fits for three of the responses are

\begin{tabular}{|l|l|l|l|}
\hline $\begin{array}{l}\text { Time } \\
\text { (hr) }\end{array}$ & Subject & G & C \\
\hline $\mathbf{0}$ & F & 0 & 0 \\
\hline $\mathbf{0 . 1 7}$ & 0 & 13.4 & 52.8 \\
\hline $\mathbf{0 . 3 3}$ & 259.9 & 64.9 & 96.8 \\
\hline $\mathbf{0 . 5}$ & 358.2 & 219.0 & 138.3 \\
\hline $\mathbf{0 . 7 5}$ & 231.9 & 304.0 & 148.6 \\
\hline $\mathbf{1}$ & 184.4 & 301.3 & 164.6 \\
\hline $\mathbf{1 . 5}$ & 155.7 & 267.9 & 143.6 \\
\hline $\mathbf{2}$ & 148.6 & 221.3 & 131.7 \\
\hline $\mathbf{2 . 5}$ & 223.5 & 253.9 & 144.4 \\
\hline $\mathbf{3}$ & 227.8 & 343.1 & 154.9 \\
\hline $\mathbf{4}$ & 245.7 & 240.6 & 166.2 \\
\hline $\mathbf{6}$ & 220.7 & 132.5 & 117.6 \\
\hline $\mathbf{8}$ & 136.9 & 90.9 & 109.1 \\
\hline $\mathbf{1 0}$ & 93.9 & 62.2 & 90.5 \\
\hline $\mathbf{1 2}$ & 70.1 & 39.6 & 80.5 \\
\hline $\mathbf{2 4}$ & 47.2 & 17.8 & 43.2 \\
\hline
\end{tabular}

Table 1: Data Set 1 - Plasma concentrations $(\mathrm{ng} / \mathrm{ml})$ for veralipride; dose $=100 \mathrm{mg}$ in $200 \mathrm{ml}$ of water. 
Citation: Godfrey KR, Arundel PA, Zhu W, Dong Z, Bryant R (2011) Modelling the Double Peak Phenomenon. J Bioequiv Availab 3: 101-107. doi:10.4172/jbb.1000068

shown in Figure 4. A logarithmic scale for the concentration has been chosen to illustrate the fact that the slope of the fit at the tail end of the data is not very accurate for two of these responses.

\begin{tabular}{|l|l|l|l|}
\hline $\begin{array}{l}\text { Time } \\
\text { (hr) }\end{array}$ & Subject & \multicolumn{2}{l|}{} \\
\hline $\mathbf{0}$ & $\mathbf{A}$ & $\mathbf{C}$ & 0 \\
\hline $\mathbf{0 . 5}$ & 160 & 0 & 13 \\
\hline $\mathbf{1}$ & 195 & 12.5 & 38 \\
\hline $\mathbf{1 . 5}$ & 130.5 & 50 & 69 \\
\hline $\mathbf{2}$ & 219.5 & 43 & 94 \\
\hline $\mathbf{2 . 5}$ & 198.5 & 175 & 94 \\
\hline $\mathbf{3}$ & 189 & 139 & 84 \\
\hline $\mathbf{3 . 5}$ & 194 & 107 & 52 \\
\hline $\mathbf{4}$ & 133.5 & 165 & 56 \\
\hline $\mathbf{5}$ & 83.5 & 187.5 & 84 \\
\hline $\mathbf{6}$ & 55.5 & 100 & 137 \\
\hline $\mathbf{8}$ & 36 & 58.5 & 54 \\
\hline $\mathbf{1 0}$ & 32 & 50 & 37 \\
\hline $\mathbf{1 2}$ & 30.5 & 28 & 22 \\
\hline $\mathbf{1 8}$ & 22 & 16.5 & 13 \\
\hline $\mathbf{3 6}$ & 14 & 10 & 7 \\
\hline
\end{tabular}

Table 2: Data Set 2 - Plasma concentrations ( $\mathrm{ng} / \mathrm{ml}$ ) for danazol; dose $=100 \mathrm{mg}$, corresponding to $30 \mathrm{~g}$ of the emulsion formulation, taken with $120 \mathrm{ml}$ of water.

\begin{tabular}{|l|l|l|}
\hline $\begin{array}{l}\text { Time } \\
\text { (hr) }\end{array}$ & Subject & A6 \\
\hline $\mathbf{0}$ & 0 & 0 \\
\hline $\mathbf{0 . 3 3}$ & 193 & 93 \\
\hline $\mathbf{0 . 6 7}$ & 352 & 620 \\
\hline $\mathbf{1}$ & 398 & 500 \\
\hline $\mathbf{1 . 5}$ & 500 & 352 \\
\hline $\mathbf{2}$ & 389 & 528 \\
\hline $\mathbf{2 . 5}$ & 352 & 1222 \\
\hline $\mathbf{3}$ & 352 & 1500 \\
\hline $\mathbf{4}$ & 806 & 1093 \\
\hline $\mathbf{6}$ & 445 & 695 \\
\hline $\mathbf{8}$ & 296 & 426 \\
\hline $\mathbf{1 0}$ & 222 & 296 \\
\hline $\mathbf{1 2}$ & 185 & 185 \\
\hline
\end{tabular}

Table 3: Data Set 3 - Plasma concentrations $(\mathrm{ng} / \mathrm{ml})$ for ranitidine; dose $=300 \mathrm{mg}$ in $240 \mathrm{ml}$ of water.

\begin{tabular}{|l|l|}
\hline Time (hr) & Subject M240 \\
\hline $\mathbf{0}$ & 0 \\
\hline $\mathbf{0 . 2 5}$ & 18.6 \\
\hline $\mathbf{0 . 5}$ & 45.4 \\
\hline $\mathbf{1}$ & 371.4 \\
\hline $\mathbf{2}$ & 693.4 \\
\hline $\mathbf{3}$ & 866 \\
\hline $\mathbf{4}$ & 793.8 \\
\hline $\mathbf{5}$ & 752.4 \\
\hline $\mathbf{6}$ & 714.4 \\
\hline $\mathbf{8}$ & 731.8 \\
\hline $\mathbf{1 2}$ & 455.4 \\
\hline $\mathbf{2 4}$ & 2903.6 \\
\hline $\mathbf{3 6}$ & 1341 \\
\hline $\mathbf{4 8}$ & 1063.6 \\
\hline
\end{tabular}

Table 4: Data Set 4 - Plasma concentrations (ng/ml) for AstraZeneca Compound $B$; dose $=20 \mathrm{mg} / \mathrm{kg}$ in $100 \mathrm{ml}$ of solution.

\begin{tabular}{|l|l|l|l|l|l|}
\hline \multicolumn{5}{|c|}{ Normalised RMS Error } \\
\hline $\begin{array}{l}\text { Data } \\
\text { Set }\end{array}$ & $\begin{array}{l}\text { Model } \rightarrow \\
\text { Subject } \downarrow\end{array}$ & VA(1) & PI(1) & VA(2) & PI(2) \\
\hline \multirow{2}{*}{$\mathbf{1}$} & F & 0.217 & 0.216 & 0.134 & $\mathbf{0 . 0 7 6}$ \\
\cline { 2 - 6 } & G & 0.068 & 0.073 & 0.067 & $\mathbf{0 . 0 6 4}$ \\
\cline { 2 - 6 } & C & 0.070 & 0.066 & $\mathbf{0 . 0 3 4}$ & 0.065 \\
\hline \multirow{2}{*}{$\mathbf{2}$} & A & $\mathbf{0 . 1 4 9}$ & 0.153 & 0.152 & 0.153 \\
\hline & B & 0.295 & 0.265 & 0.299 & $\mathbf{0 . 2 0 4}$ \\
\hline & C & 0.167 & 0.148 & $\mathbf{0 . 0 9 0}$ & 0.135 \\
\hline \multirow{2}{*}{$\mathbf{3}$} & A2 & 0.146 & 0.144 & 0.146 & $\mathbf{0 . 1 3 1}$ \\
\hline & A6 & 0.138 & $\mathbf{0 . 0 9 0}$ & 0.114 & 0.104 \\
\hline \multirow{2}{*}{$\mathbf{4}$} & & & & & \\
\hline
\end{tabular}

Table 5: Comparison of Normalised RMS Errors - unweighted data, for subjects from Tables $1-4$ using four models as specified, heavy type shows the minimum value for each row.

\begin{tabular}{|l|l|l|l|l|l|}
\hline \multicolumn{5}{|c|}{ Normalised RMS Error } \\
\hline $\begin{array}{l}\text { Data } \\
\text { Set }\end{array}$ & $\begin{array}{l}\text { Model } \rightarrow \\
\text { Subject } \downarrow\end{array}$ & VA(1) & PI(1) & VA(2) & PI(2) \\
\hline \multirow{2}{*}{$\mathbf{1}$} & F & 0.092 & 0.093 & 0.053 & $\mathbf{0 . 0 4 8}$ \\
\hline & G & 0.084 & 0.085 & 0.079 & $\mathbf{0 . 0 2 6}$ \\
\hline & C & 0.036 & 0.036 & $\mathbf{0 . 0 2 7}$ & 0.036 \\
\hline & & & & & \\
\hline \multirow{2}{*}{$\mathbf{2}$} & A & 0.135 & 0.136 & 0.132 & $\mathbf{0 . 0 4 7}$ \\
\cline { 2 - 6 } & B & 0.157 & 0.150 & 0.159 & $\mathbf{0 . 0 7 7}$ \\
\hline & C & 0.141 & 0.136 & $\mathbf{0 . 0 3 2}$ & 0.133 \\
\hline \multirow{2}{*}{$\mathbf{3}$} & A2 & 0.071 & 0.073 & 0.070 & $\mathbf{0 . 0 3 6}$ \\
\hline & A6 & 0.058 & 0.058 & 0.053 & $\mathbf{0 . 0 4 6}$ \\
\hline $\mathbf{4}$ & & & & & \\
\hline
\end{tabular}

Table 6: Comparison of Normalised RMS Errors - weighted data, for subjects from Tables $1-4$ using four models as specified, heavy type shows the minimum for each row.

To determine whether the fitting of the tail end of the data could be improved, a further set of model fits was made with weighted data. As there is no simple closed-form solution of the equations for either approach Berkeley Madonna is used to solve numerically the differential equations (1) and (2) for the Variability of Absorption models and (3) and (3A) for the Parallel Inputs models. In this situation it is not possible to directly weight the least-squares errors, between model-predicted and actual data, thus a different approach is used. The weighting on the last data point was introduced empirically by adding fifty identical values at very small time increments below the time of the last data point and fifty above it, so weighting the last data point by 101. The weighting on the two preceding data points was done similarly with five points below the time of the data point and five above it, so weighting both these points by 11 . The results are shown in Table 6 and the weighted data fits for the same three responses as in Figure 4 are shown in Figure 5. The precise choice of weighting on these last three data points was made to provide a close fit to the tail end of the data for all the data sets, while at the same time avoiding a significantly worse fit to the earlier data points.

The fits to the response of Data Set 4 are shown in Figure 6; the unweighted and weighted data fits being shown together in this Figure to emphasise how weighting can result in a higher RMS Error. 
Citation: Godfrey KR, Arundel PA, Zhu W, Dong Z, Bryant R (2011) Modelling the Double Peak Phenomenon. J Bioequiv Availab 3: 101-107. doi:10.4172/jbb.1000068

\section{Discussion}

\section{The following points emerge from inspection of the results}

- From Tables 5 and 6 , one of the 2-compartment fits has given the lowest RMS Error for most of the responses both with unweighted data and with weighted data.

- From Table 5, it can be seen that all four models give very similar RMS Error for Data Set 2, Subject A, with unweighted data.

- From Tables 5 and 6, the lowest RMS Error with both unweighted and weighted data has been obtained in the majority of cases using Model PI(2).

- From Tables 5 and 6, the minimum RMS Error using weighted data is reduced, in several cases considerably, compared with the corresponding fits using unweighted data for all the responses except two. The exceptions are Data Set 1, Subject $\mathrm{C}$, which was included in the study to see whether the two new methods described in this paper would result in lower RMS Error for a response whose tail end was already fitted well with unweighted data, and Data Set 4.

- From Figures $4 \mathrm{~A}$ and $5 \mathrm{~A}$ and Figures $4 \mathrm{~B}$ and 5B, the weighting of the data greatly improves the estimation of the slope at the tail end of the data for Data Set 1, Subject G and Data Set 2, Subject C, which would in turn give a much better estimate of the terminal-phase half-life.

- From Figures $4 \mathrm{C}$ and $5 \mathrm{C}$, the visual improvement when weighting the data in the fitting of the tail end of the data for
Data Set 3, Subject A2, is comparatively modest. In the light of this, the margin of improvement in least RMS Error for this response is perhaps a little surprising, given that the fit was already good for the unweighted data (Figure 4C).

- From Tables 5 and 6, it can be seen that for Data Set 4, the lowest Normalised RMS Error is 0.120 for the unweighted data (Model VA(2)), and 0.147 for the weighted data (Model VA(1) or $\mathrm{VA}(2))$. The reason in this case is that the last data point is at $t=48$ hours, and that at this time, the concentration value is still quite high - in other words, the tail end of the data has not yet been reached. The best fits, shown in Figure 6, indicate that while weighting improves the fit of the last data point, this is at the expense of less close fits at the earlier data points.

In conclusion the advantage of the Variability of Absorption approach is that it has a clear physiological interpretation, with zero rate of absorption assumed from the jejunum, although the assumption of constant input rates over the two other zones, corresponding to the duodenum and the ileum, is an idealization. The drawback is that it is essential to try a very large number of initial guesses for the model parameters, to be reasonably confident of reaching the global minimum.

The advantage of the Parallel Inputs approach is that there are considerably fewer parameters to be estimated, and as a consequence, the estimation is far less critical to the initial parameter guesses, converging to the same final values from a relatively large range of these. The drawback now is that there is no direct physiological interpretation, although the impulsive dosing is realistic. As applied here, the method is also time-consuming, because numerous combinations of the
(4A) Data Set 1, Subject G, Model PI(2)

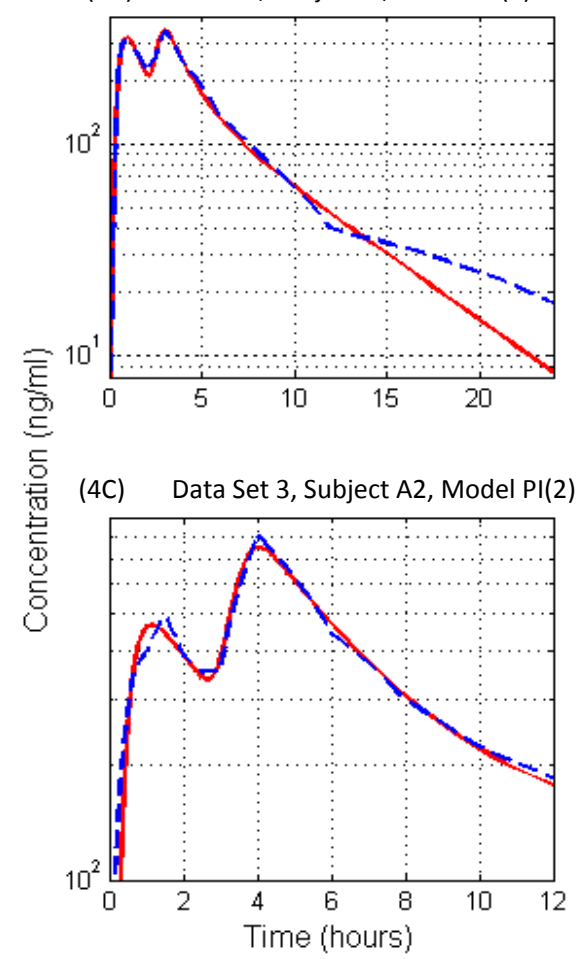

(4B) Data Set 2, Subject C, Model VA(2)
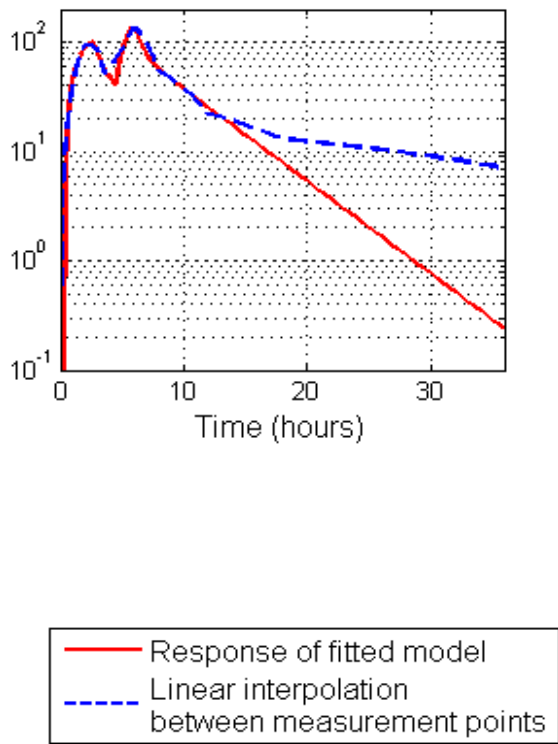

Figure 4: Best model fits with unweighted data 
Citation: Godfrey KR, Arundel PA, Zhu W, Dong Z, Bryant R (2011) Modelling the Double Peak Phenomenon. J Bioequiv Availab 3: 101-107. doi:10.4172/jbb.1000068

(a) Data Set 3, Subject G model Pl(2)

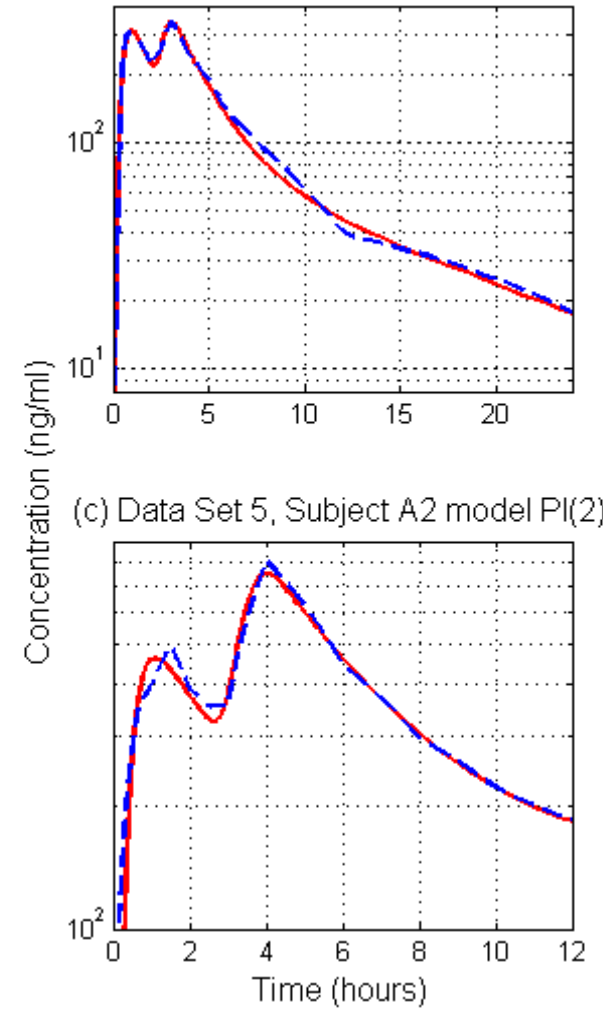

(b) Data Set 4, Subject C model VA(2)

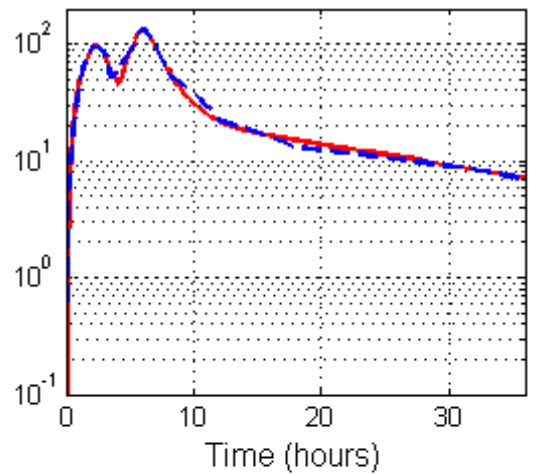

- Response of fitted model Linear interpolation between measurement points

Figure 5: Best model fits with weighted data.

number of compartments in each pathway have to be tried. Overall, it takes approximately the same amount of time to apply either of the two approaches.

To improve the fit to the tail-end of the data two methods were tried. First, the performance of the Parallel Inputs approach with

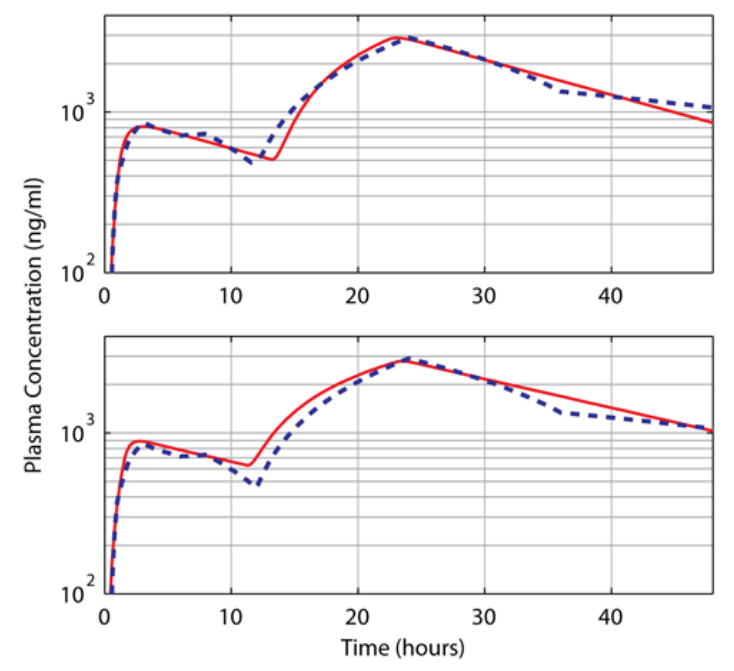

Figure 6: Best model fits for Data Set 4, with (upper) unweighted data (Model VA(2)) and (lower) weighted data (Model VA(1)); dashed line: linear interpolation between measurement points; continuous line: response of fitted model. two compartments to model the distribution and elimination of the drug was investigated. Second, the possibility of obtaining better fits by weighting the last three concentration values in the response was examined.

The lowest RMS Error for these seven responses was obtained using Model PI(2) in six cases and Model VA(2) in the remaining case. This illustrates the importance of using both methods of modelling.

For the response of Data Set 4, the RMS values were again similar for all the models for the unweighted data, but this time, weighting noticeably increased the RMS Error values. It can be seen from Figure 6 that while the tail end of the response is fitted more closely, it is at the expense of a less close fit earlier in the response.

It was found that in nearly all cases remarkably good fits to the data could be obtained using a particular combination of model and/ or weighting. It is therefore definitely worthwhile having both methods of modelling to hand. However it is essential to examine the curve fits graphically, rather than just relying on RMS Error values.

The modelling techniques described here can also be applied to many of the compounds detailed in the extensive review of multiple peaking by Davies et al. [3].

\section{Acknowledgements}

The work described was the topic of the M.Sc. Projects of two of the authors (Zhu and Dong) and of the Final Year Undergraduate Project of another of the authors (Bryant) while they were students in the School of Engineering at the University of Warwick. These Projects were jointly supervised by the other two authors (Godfrey and Arundel). As such, there was no external funding, and there are no conflicts of interest. 
Citation: Godfrey KR, Arundel PA, Zhu W, Dong Z, Bryant R (2011) Modelling the Double Peak Phenomenon. J Bioequiv Availab 3: 101-107. doi:10.4172/jbb.1000068

\section{References}

1. Berkeley Madonna - Modeling and Analysis of Dynamic Systems (2008), http:// www.berkeleymadonna.com/ [9 April 2009]

2. Charman WN, Rogge MC, Boddy AW, Barr WH, Berger BM (1993) Absorption of danazol after administration to different sites of the gastrointestinal tract and the relationship to single- and double-peak phenomena in the plasma profiles. J Clin Pharmacol 33: 1207-1213.

3. Davies NM, Takemoto JK, Brocks DR, Yanez JA (2010) Multiple Peaking Phenomena in Pharmacokinetic Disposition. Clin Pharmacokinet 49: 351-377.

4. Godfrey KR, Arundel PA, Dong Z, Bryant R (2010) Modelling the double peak phenomenon. Comput Methods Programs Biomed, On-line DOI: 10.1016/j. cmpb.2010.03.007.

5. Jacquez JA (1996) Compartmental Analysis in Biology and Medicine Biomedware, Ann Arbor, MI.

6. Lennernäs H and Regårdh CG (1993) Evidence for an interaction between the $\beta$-blocker pafenolol and bile salts in the intestinal lumen of the rat leading to dose-dependent oral absorption and double peaks in the plasma concentrationtime profile. Pharma Res 10: 879-883.

7. Marathe PH, Sandefer EP, Kollia GE, Greene DS, Barbhaiya RH, et al. (1998) In vivo evaluation of the absorption and gastrointestinal transit of avitriptan in fed and fasted subjects using gamma scintigraphy. Journal of Pharmacokinet Biopharm 26: 1-20

8. Metsugi Y, Miyaji Y, Ogawara KI, Higaki K, Kimura T (2008) Appearance of double peaks in plasma concentration-time profile after oral administration depends on gastric emptying profile and weight function. Pharm Res 25: 886-895.
9. Mirfazaelian A, Mahmoudian M (2006) A simple pharmacokinetics subroutine for modeling double peak phenomenon. Biopharm Drug Dispos 27: 119-124.

10. Oberle RL and Amidon GL (1987) The influence of variable gastric emptying and intestinal transit rates on the plasma level curve of cimetidine; an explanation for the double peak phenomenom. J Pharmacokinet Biopharm 15: 529-544.

11. Ogiso T, Kasutani M, Tanaka H, Iwaki M and Tanino T (2001) Pharmacokinetics of epinastine and a possible mechanism for double peaks in oral plasma concentration profiles. Biol Pharm Bull 24: 790-794.

12. Okuyasana O, Forrest A, DiFrancesco R, Bilic S, Rosenkranz S, et al. (2007) Compartmental analysis of oral amprenavir with secondary peaks. Antimicrob Agents Chemother 51: 1822-1826.

13. Piquette-Miller M, Jamali $F$ (1997) Pharmacokinetics and multiple peaking of acebutolol enantiomers in rats. Biopharm Drug Dispos 18: 543-556.

14. Plusquellec Y, Campistron G, Staveris S, Barre J, Jung L, et al. (1987) A double-peak phenomenon in the pharmacokinetics of veralipride after oral administration: a double-site model for drug absorption. J Pharmacokinet Biopharm15: 225-239

15. Wermeling DP, Miller JL, Archer SM, Manaligod JM, Rudy RC (2001) Bioavailability and pharmacokinetics of lorazepam after intranasal, intravenous, and intramuscular administration. J Clin Pharmacol 41: 1225-1231.

16. Yin OQ, Tomlinson B, Chow AH, Chow MS (2003) A modified two-portion absorption model to describe double-peak absorption profiles of ranitidine. Clin Pharmacokinet 42: 179-192. 